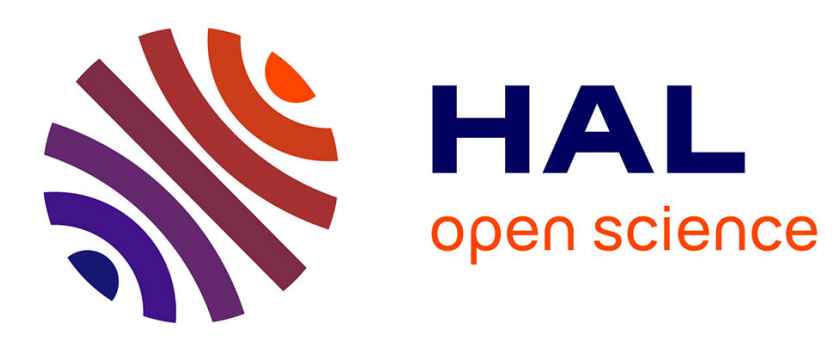

\title{
Uncertainty on gringe projection technique: A Monte-Carlo-based approach
}

\author{
Jérôme Molimard, Laurent Navarro
}

\section{To cite this version:}

Jérôme Molimard, Laurent Navarro. Uncertainty on gringe projection technique: A MonteCarlo-based approach. Optics and Lasers in Engineering, 2013, 51 (7), pp.840-847. 10.1016/j.optlaseng.2013.01.023 . hal-00834909

\section{HAL Id: hal-00834909 https://hal.science/hal-00834909}

Submitted on 19 Jun 2013

HAL is a multi-disciplinary open access archive for the deposit and dissemination of scientific research documents, whether they are published or not. The documents may come from teaching and research institutions in France or abroad, or from public or private research centers.
L'archive ouverte pluridisciplinaire HAL, est destinée au dépôt et à la diffusion de documents scientifiques de niveau recherche, publiés ou non, émanant des établissements d'enseignement et de recherche français ou étrangers, des laboratoires publics ou privés. 


\section{Uncertainty on fringe projection technique: a Monte-Carlo-based approach}

LCG, UMR 5146,

\section{Abstract}

7 Error estimation on optical full field techniques (OFFT) is millstone in the diffusion of OFFT.

8 The present work describes a generic way to estimate overall error in fringe projection, either

9 due to random sources (phase error, basically related to the quality of the camera and of the

10 fringe extraction algorithm) or the bias (calibration errors). Here, a high level calibration

11 procedure based on pinhole model has been implemented. This model compensates for the

12 divergence effects of both the video-projector and the camera. The work is based on a Monte

13 Carlo procedure. So far, the complete models of the calibration procedure and of a reference

14 experiment are necessary. Here, the reference experiment consists in multiple step out-of-

15 plane displacement of a plane surface. Main conclusions of this work are: $1 /$ the uncertainties

16 in the calibration procedure lead to a global rotation of the plane, $2 /$ the overall error has been

17 calculated in two situations; the overall error ranges from $104 \mu \mathrm{m}$ down to $10 \mu \mathrm{m}, 3 /$ the main

18 error source is the phase error even if errors due to the calibration are not always negligible.

20 Keywords: Fringe projection; Uncertainty analysis; Monte Carlo error propagation

\section{1. Introduction}

22 Optical full field techniques (OFFT) are nowadays common tools in university laboratories. 
1 Anyway, the confidence on the result obtained is poorly described, and error estimation on

2 OFFT is millstone in their diffusion in industrial world. Usually, the measuring chain is

3 complex, implying optical elements, numerical processing (correlation, phase extraction ...)

4 and post-processing (derivation, filtering ...). A lot of work has been carried out in order to

5 improve and/or characterize each element of the measuring chain, in particular for image

6 correlation [1] [2] or phase extraction [3]. Again, some experimental work gives a global sight

7 on errors, see for example [4] or [5]. Some work also was done in order to reduce phase errors

8 (see for example [6]). Anyway, overall measurement error still never has been achieved, in

9 particular because of the difficulties to integrate different error sources, among them errors

10 due calibration procedure. Prediction through error model is not straightforward and usually

11 cannot be achieved using standard error propagation rules. Previous works show the

12 efficiency of Monte-Carlo based procedure on specific element of the measuring chain.

13 Description of the error on phase extraction has been provided by Cordero [7]; post-

14 processing derivation has been investigated in the same way [8]. Beside these two general

15 purpose works, a study on 3D ESPI leads to an optimal position of illumination vectors [9].

16 Anyway, no global prediction approach has been carried out to the best of our knowledge.

17 Among the different OFFTs, fringe projection is one of the more spread, since its first 18 development [10 12]. Basically, the method renders a shape [5] or a shape variation [13].

19 Coupled with a 2D correlation system, it can be extended to the measurement of any

20 displacement of a non-flat surface $[14,15,16]$. Since it is a non-contacting method, a lot of

21 applications are developed or under development in health engineering (see for example [17,

$2218,19])$.

23 The present work describes a generic way to estimate overall error in fringe projection, either

24 due to random sources (phase error, basically related to the quality of the camera and of the

25 fringe extraction algorithm) or the bias (calibration errors). Here, a high level calibration 
1 procedure based on pinhole model has been implemented [18]. This model compensates for

2 the divergence effects of both the video-projector and the camera. The Monte Carlo procedure

3 requires complete models of the calibration procedure and of the reference experiment. Here,

4 the reference experiment consists in multiple steps out-of-plane displacement of a plane

5 surface. In order to give boundary values to the overall error, two different situations are

6 investigated: the first one is common macroscopic fringe projection set-up. The second one is

7 a microscopic set-up, optimized for random noise for example considering a larger set of

8 images in the phase extraction.

9 The paper presents first the Monte-Carlo procedure; then, the specific fringe projection

10 approach is described. Last, the implementation for a given set of experimental conditions is

11 developed, results are analyzed.

\section{2. Monte Carlo based uncertainty approach}

14 The uncertainty associated with the result of a measurement is a parameter that characterizes

15 the dispersion of values that can reasonably be attributed to the measurand. Operationally, the

16 dispersion of values of some quantity $Q$ is described by a probability density function (PDF),

$17 f(Q)$. The domain of the PDF consists of all possible values of $Q$, and its range is in the

18 interval $(0,1)$. If the PDF is known, the estimate of $Q$ is obtained by evaluating the expected

19 value and its standard uncertainty is taken to be equal to the standard deviation [25].

20 Although obtaining the most appropriate PDF for a particular application is not

21 straightforward, if the measurand $Q$ is related to a set of other quantities $P=\left(P_{1} \ldots P_{n_{P}}\right)^{T}$

22 through a measurement model $Q=M(P)$, linear or weakly non-linear, the standard

23 uncertainty of $Q$ can be expressed in terms of the standard uncertainties of the input quantities 
$1 \quad\left(P_{1} \ldots P_{n_{P}}\right)$ by using the so-called law of propagation of uncertainties (LPU) [25, 26]. Instead

2 of the LPU, a Monte Carlo-based technique [22-24] can be applied to linear as well as to

3 nonlinear models, on independent or co-varying error sources.

4 The Monte Carlo-based technique requires first assigning Probability Density Functions

5 (PDFs) to each input quantity. Next, a computer algorithm is set up to generate an input

6 vector $p_{1}=\left(p_{1} \ldots p_{n_{P}}\right)^{T}$; each element $p_{j}$ of this vector is generated according to the specific

7 PDF assigned to the corresponding quantity $P_{j}$. By applying the generated vector $p_{1}$ to the

8 model $Q=M(P)$, the corresponding output value $q_{1}$ can be computed. If the simulating

9 process is repeated $N$ times $(N>>1)$, the outcome is a series of indications $\left(q_{1} \ldots q_{N}\right)^{t}$ whose

10 frequency distribution allows us to identify the PDF of $Q, f(q)$. Then, irrespective of the form

11 of this PDF, the estimate $q_{e}$ and its associated standard uncertainty $u\left(q_{e}\right)$ can be calculated

12 by

$$
q_{e}=\frac{1}{N} \sum_{l=1}^{N} q_{l}
$$

14 and

$$
u\left(q_{e}\right)=\left(\frac{1}{(N-1)} \sum_{l=1}^{N}\left(q_{l}-q_{e}\right)^{2}\right)^{1 / 2}
$$

16 Knowledge of each element of the $P$ vector, in particular the uncertainty level and the PDF

17 shape, directly derives from the experimental knowledge. So far, a good understanding of the

18 whole set-up and procedure is necessary. Here, we suppose that each error source is

19 independent; anyway, cross-dependent inputs are possible.

\section{3. The pin-hole Model}

21 The classical pin-hole model characterizes the geometrical relationship between a point in 3D 
1 space and its projection on a plane behind another plane in which an aperture was performed.

2 This aperture is supposed to be a point (hence the name pinhole). The figure 1 illustrates the

3 principle of the pin-hole model in two dimensions, as the 3D extrapolation is quite simple.

$4 \quad O$ is the aperture and $Y$ is the plane in which the aperture was performed, $P$ is the point in

$53 \mathrm{D}$ space, $x_{p}$ and $y_{p}$ its coordinate. $Q$ is the projection of $P$ in the projection plane $Y^{\prime}$,

$6 f$ and $y_{q}$ are its coordinates. Then, the simple equation $y_{q}=-f \frac{y_{p}}{x_{p}}$ describes the relationship

7 between a point $P$ in 3D space and its projection $Q$ in 2D plane. The dotted line is called the

8 projection line. This model is generally used in shape / displacement measurement systems to

9 account for perspective effects, either for fringe projection [18] [27] or stereo-correlation [28].

10 Note anyway that the following work takes into account perspective effects with an

11 assumption of negligible distortions. In the same way, the optical model does not take into

12 account off-axis arrangement that should be found in many video-projectors. These two points

13 can be considered as the main limitations of the presented work; anyway, the material used in

14 the following is chosen under these hypothesis: dedicated low-distortion lenses, and an in line

15 video-projector.

16

17 4. 3D surface implementation

18 4.1. PRINCIPLE OF FRINGE PROJECTION

19 The fringe projection method has already been described by many authors [13, 16, 17, 21].

20 The physical principle is straightforward: a periodic pattern is projected on an object; the light

21 is diffused by the object and captured by a CCD video-camera. The deformation of the

22 fringes, recorded as phase maps, has a known dependency to the shape of the illuminated 
1 object.

2 Since the fringe projection technique uses the light diffused by an object in order to measure

3 its shape or shape variation, a surface preparation consisting usually in a white paint is

4 sometimes useful. Moreover, in order to observe out-of-plane displacements, the angle

5 between the projected fringes and the observed diffused light must not be null (fig. 3). Light

6 intensities on an object illuminated by a set of fringes can be described by a periodic function

$7 I_{l i}$, with a perturbation $\varphi$ corresponding to the object shape:

8

$$
I_{l i}(x, y)=I_{0}(x, y)\left[1+\mathrm{Y}(x, y) \times \cos \left(\frac{2 \pi}{p(x, y)} y+\phi(x, y)\right)\right]
$$

10 This equation involves an average intensity $I_{0}$ and a contrast $\gamma$. These values should be 11 constant over the whole map, but some low-frequency variations due to illumination 12 inhomogeneity or diffusivity changes on top of the surface can occur. Consequently, both 13 average intensity and contrast have to be considered as local quantities and can be denoted $14 I_{0}(x, y)$ and $\gamma(x, y)$. The pitch, $p$ is the distance between two light peaks on a flat surface i.e. a 15 period of the cosine function in the ideal case. Again, due to perspective effects in particular, 16 this pitch can change over the map, but this variation can be known either using a model or a 17 calibration procedure. Last, the object is responsible for a phase shift $\varphi=\varphi(x, y)$ at each point 18 of the field, as expressed by:

$$
\phi(x, y)=\frac{2 \pi \times \tan \theta(x, y)}{p(x, y)} z(x, y)
$$

22 In this expression, the sensitivity characterized by the slope of the linear relationship between 
$1 \varphi(x, y)$ and $z(x, y)$, can be adjusted by modifying the pitch $p$ or the angle $\theta$ between the CCD

2 video-camera and the video-projector. Again, it has to be noted that the sensitivity can vary

3 locally. In particular, the video projector and the CCD camera commonly use divergent lens.

4 Since the sensitivity usually varies within the measuring area, a more complete model has to

5 be used; here, the pin-hole model is chosen because it is simple and therefore open to 6 interpretation.

\section{4.2. APPLICATION OF THE PIN-HOLE MODEL}

8 The classical pin-hole model is well adapted to such a configuration. Parameters of the model 9 are:

10 - the camera magnification along the vertical axis $\left(\gamma_{C C D}\right)$ and along the horizontal axis

$11 \quad \frac{\gamma_{C C D}}{\tau_{C C D}}$

12 - the distance between the CCD camera and the reference plane $\left(h_{0}\right)$,

13 - the distance between the video-projector and the reference plane $\left(h_{p}\right)$,

14 - the distance between the video-projector focal point and the CCD camera axis $(d)$.

16 Measuring all these parameters is difficult in practice and an inverse calibration is more

17 adapted. Here, the calibration is based on the known rotation of a reference plane [18].

18

19 Now, application of the pin-hole model gives the following set of equations: 20 
$1 z(r, s)=\frac{h_{p} h_{o}\left[\left(2 \pi f_{p} h_{p}-P_{t} d \phi\right) \frac{\mathrm{Y}_{C C D}}{\mathrm{~T}_{C C D}} \times r-P_{t}\left(d^{2}+h_{p}^{2}\right) \phi(r, s)\right]}{h_{0}\left[\left(2 \pi f_{p} h_{p}-P_{t} d \phi\right) d+P_{t}\left(d^{2}+h_{p}^{2}\right) \phi(r, s)\right]-h_{p}\left[2 \pi f_{p} h_{p}-P_{t} d \phi(r, s)\right] \frac{\mathrm{Y}_{C C D}}{\mathrm{~T}_{C C D}} \times r}$

$$
x(r, s)=\frac{z(r, s)+h_{0}}{h_{0}} \frac{\mathrm{Y}_{C C D}}{\mathrm{~T}_{C C D}} \times r
$$

$$
y(r, s)=\frac{z(r, s)+h_{0}}{h_{0}} \gamma_{C C D} \times s
$$

4 The point $A(x, y, z)$ is known for any position in the object plane, referred by the coordinates

$5 M(r, s)$. Note that $x$ and $y$ coordinates don't correspond to the $\left(\frac{\mathrm{V}_{C C D}}{\mathrm{~T}_{C C D}} \times r, \mathrm{Y}_{C C D} \times s\right)^{t}$ because

6 of the perspective effect on the camera.

\section{4.3. PHASE EXTRACTION}

8 Extraction of the phase from intensity map(s) requires either spatial or temporal phase shifting

9 techniques. The Photomecanix software, developed in the laboratory, has genuine

10 implementation of both techniques, as prescribed by Surrel [29]. The choice only depends on

11 the situation: if temporal effects are expected, spatial phase shifting is more appropriate,

12 because it only requires one image [30]. If not, temporal phase shifting technique should be

13 preferred for its higher spatial resolution [13]. Only this method is briefly described here.

14 A set of $\mathrm{n} \times \mathrm{q}$ fringe patterns with a known phase shift $q / 2 \pi$ is projected successively on the

15 surface, first and last fringe pattern being shifted by a $n \times 2 \pi, n \in$ ? phase. Then, the intensity

16 variation at each point (i.e. each camera pixel) corresponds to a sine wave function with an

17 initial phase shift. The phase is evaluated using the Fourier Transform: 


$$
\phi(r, s)=\arctan _{2 \pi}\left(\frac{\sum_{k=1}^{n q}\left\{\sin \left(k \square \frac{2 \pi}{q}\right) I_{k}(r, s)\right\}}{\sum_{k=1}^{n q}\left\{\cos \left(k \square \frac{2 \pi}{q}\right) I_{k}(r, s)\right\}}\right)
$$

3 This implementation is based on a sine-wave variation of the projected light. Actually, the

4 video-projector or the camera has a non-linear response; so far, the recorded signal exhibits

5 some harmonics due to this non-linearity. Surrel has proved that the implementation he

6 proposed minimizes the harmonics effect [29]; in practice, harmonics might have amplitude

7 similar to the random noise, and do not present a specific and significant error source: in the

8 error propagation model, these two effects will be represented by the same parameter. Indeed,

9 some recent works tend to minimize these harmonics effects, see for example [6].

10 Metrological performances of the shape measurement set-up are interesting compared to the

11 classical stereovision technique: the spatial resolution is 1 pixel ( 8 to $156 \mu \mathrm{m}$, depending on

12 the field of view), and the typical resolution ranges from $\sigma=0.5$ to 1 hundredth of fringe, i.e.

$133 \mu \mathrm{m}$ at best. This capacity is very important for high frequency phenomena monitoring: skin

14 submitted to mechanical load [17, 19], cuticle sleeves [20], ..

16 5. Experimental set-up and performances

\section{$17 \quad$ 5.1. OPTICAL TEST-RIG}

18 The optical set-up for 3D measurement is a classical fringe projection set-up, with a pocket-

19 projector $3 \mathrm{M}$ MPRO 110 of $640 \times 480$ pixels resolution and an 8 bits CCD camera Imaging 
1 Source of $1280 \times 960$ pixels resolution. This solution is adapted to fields of investigation from

$210 \times 7 \mathrm{~mm}^{2}$ to $200 \times 150 \mathrm{~mm}^{2}$ (see figure 2).

3 The system uses a low-distortion lens (Linos, $0.3 \times \mathrm{f} / 8$ ). The evaluation of the distortion using

4 Bouquet algorithm [31] shows that the error related to this parameter is very low (less than

$510^{-4}$ ). In the following, this error will not be considered for the sake of simplicity. The

6 selected video-projector has no lens offset. Such an offset would result in a vertical

7 translation of the optical axis; a global uncertainty range for the vertical translation is

8 proposed hereafter.

\section{5.2. CALIBRATION PROCEDURE}

10 The calibration procedure is divided into two steps: first, the phase map of a plane

11 perpendicular to the camera axis is taken. Second, the plane is rotated along the vertical axis,

12 and a second phase map is recorded. Even if the method is straightforward, some hypothesis

13 should be fulfilled: video-projector and camera axis should converge on a single point, this

14 point being on the rotation axis; rotation axis should be perpendicular to the plane defined by

15 the camera axis and the video-projector axis, and parallel to the fringes (Figure 2).

16 A complete strategy has been established to fulfill these requirements: the camera and the

17 video-projector are mounted on translation and rotation stages, allowing fine adjustments. The

18 camera is set in a Galilean frame of reference using a spirit level. The reference plane is put at

19 the desired distance; the position perpendicular to the camera is obtained using spirit level and

20 distance measurement using reference points on the camera and on the plane. Last, the video-

21 projector position is adjusted using a projected cross and a reference cross inserted in the

22 image (Figure 4). The centers of the cross materialize the optical axis respectively of video-

23 projector and of the camera, and both centers have to be superposed. The vertical and 
1 horizontal lines make visible the horizontal and the vertical axis of each frame of reference.

2 Again, these lines have to be superposed.

3 This implementation is verified after completing a first calibration by analyzing the shape of

4 each plane: at the reference position, the tilting of the plane can be evaluated. After rotation,

5 the difference between the two positions indicates the verticality of the rotation axis and its

6 position compared to the camera frame of reference. The implementation is independent of

7 the position of the rotation axis, but it is better to center it in order to obtain a symmetric

8 calibrated volume. Finally, it is then possible to have an experimental estimate of the plane

9 tilting, and rotation axis; the calibration is validated if the tilting of the plane or the rotation

10 axis is lower than $1 / 10$ th of millimeter, this value being a minimum adjustable value

11 considering the set-up.

12 Last, the phase quality can be estimated by comparing the theoretical phase surface to the

13 experimental one. In the particular case shown Figure 5/a, the phase error is strongly affected

14 by harmonics due to a non-sinusoidal fringe intensity shape. This situation is usually rejected,

15 and illumination is more carefully tuned, but it is a didactic example to show the fringe

16 correction implemented: because the phase error due to harmonics is deterministic, it can be

17 compensated using a look-up table, see Figure 5/b. In this last situation, the main part of the

18 phase error is cancelled, with some low-frequency fluctuations related to the reference

19 surface. Now, an analysis on the error distribution (Figure 5/c and 5/d) shows that the phase

20 error can be modeled as a random Gaussian distribution at a global level. Moment normality

21 tests are positive for each situation; residue normality test is positive only for the corrected

22 phase map.

23 Note that the system has to be calibrated after each geometrical change in the configuration,

24 but not before each new experiment. 


\section{5.3. SHAPE MEASUREMENT}

2 A reference experimental test has been specially designed: it consists of a sphere cut in a

3 plate. The system has a standard macroscopic design, with no special optimization for this

4 specimen. Result is presented Figure 6.

5 The depression in the plate has been estimated using least square approximations of a sphere

6 and of a plate. The deepness is defined by the length between the lower sphere point and its

7 projection on the plate. Because of averaging effects due to the high number of measurement

8 points, this value becomes almost insensitive to noise. It has been measured to $2.10 \mathrm{~mm}$ and

9 the sphere radius to $15.17 \mathrm{~mm}$. High frequency component of the experimental field is used

10 to estimate random phase noise. Its standard deviation is representative of the common values

11 encountered in the laboratory $(43 \mu \mathrm{m})$. A comparison between the results obtained for the

12 sphere deepness and a dial indicator is given Table 1 . The dial indicator has a resolution of 10 $13 \mu \mathrm{m}$, so a difference in height has a resolution of $14 \mu \mathrm{m}$. Difference between fringe projection

14 and the dial indicator is $107 \mu \mathrm{m}$. This value can be related to some systematic errors, in

15 particular due to the calibration procedure, but also errors during the measurements using the

16 dial indicator.

17

18 6. Measurement models and error analysis

19 6.1. SIMULATED CONDITIONS

20 Calibration of the system is realized with the help of a white rectangular plate (figure 2).

21 Calibration procedure used in this document is based on the following simplifying

22 assumptions: 
1 1- each optical axis converges in the middle of the plate,

2 2- plate rotation axis is located at the surface of this plate, passing through the convergence

3 axis,

4 3- plate rotation is perpendicular to the optical axis planes,

5 4- rotation angle is perfectly defined.

6 Of course, in real conditions, these assumptions are not completely true, and errors on these

7 assumptions should be taken into account in order to evaluate the global uncertainty level on

8 the fringe projection process. Other error sources are related to the intensity measurement and

9 the phase extraction [7]. These errors are summarized as a random error on the phase

10 measurement. Last, camera lens distortion is not added to the model because it should become

11 too complex regarding the influence of the optical arrangement on the out-of-plane

12 information $(z)$ (see paragraph 5. ).

13 Within the Monte-Carlo framework, it is necessary to model the optical system, including its

14 possible defects, and to give a probability density function (PDF) for each error source. In

15 order to achieve such a goal, the approach will take into account the whole calibration

16 procedure, giving an estimate of the calibration parameters and, later, on the $z(x, y)$ function.

17 The measuring system model implemented for this Monte-Carlo approach will consider the

18 following uncertainties: the position of the reference plane, a random additive phase noise, an

19 error on apparent pixel size, and an error on the rotation value.

20 In order to have a good comparison on the different situations, some experimental data are

21 necessary to give a ground truth. A reference situation, corresponding to the laboratory

22 practice is defined. The field of view is $68 \times 54 \mathrm{~mm}^{2}$ and the sensitivity set to $5 \mathrm{~mm}$ per fringe.

23 Resolution is supposed to be $1 / 100^{\text {th }}$ fringe, i.e. $50 \mu \mathrm{m}$. Corresponding geometrical data are 
1 given in Table 2; the input positioning error is set to $2 \mathrm{~mm}$ for each geometrical parameter,

2 corresponding to a relative error around $1 \%$ (see Table 2). All calibration data - including

3 PDFs - are summarized in Table 3. The values are evaluated from laboratory experience.

4 Study will be held $1 /$ considering independently each error source $2 /$ using independent 5 sources all together. Each case study uses 40 random samples, giving a compromise between 6 calculation time and precision.

\section{6.2. SHAPE UNCERTAINTY}

9 In order to evaluate the influence of calibration procedure on shape reconstruction, a very

10 simple test is proposed: it consists in reconstructing the $z=0 \mathrm{~mm}$ plane, translating it and

11 reconstructing it at the position $z=1 \mathrm{~mm}$ and $z=2 \mathrm{~mm}$. The exact shape is completely

12 known, the shape variation as well.

14 Analysis of the results is based first on the bias i.e. the mismatch between the mean and the 15 expected value. Figure 7 shows the bias on the reconstruction of plane $z=0 \mathrm{~mm}$ for different 16 rotation angle, in absence of any kind of uncertainty (reference), with each uncertainty source

17 (rotation angle, reference plane angle, reference plane translation, magnification) and with the 18 conjunction of all the uncertainty sources (denoted "Total"). Results show that the mean 19 position is very close to the theoretical one in any conditions: bias error is found to be close to

$202 \times 10^{-7} \mathrm{~mm}$. It is worth noting that the global error is lower than certain individual errors.

21 This shows compensation effects between the different error sources. Surprisingly, the global

22 error level seems to be independent of the rotation angle. Addition test cases up to $15^{\circ}$ show

23 the same trends; this value is a high limit regarding the practical difficulties on using such 
1 angles. Figure 8 indicates that the standard deviation is considerably higher $\left(3 \times 10^{-4} \mathrm{~m}\right)$.

2 The values estimated on the $z=1 \mathrm{~mm}$ and $z=2 \mathrm{~mm}$ planes are the same. The shape variation

3 has a better quality anyway: the order of magnitude of the bias is the same, but the standard

4 deviation is significantly lower $\left(7 \times 10^{-5} \mathrm{~mm}\right)$. A simple explanation can be proposed: when

5 performing a differential measurement, the same calibration coefficients are used, and some

6 compensation effects exist. The analysis of reconstructed maps clearly shows that the standard

7 deviation amplitude on shape maps is due to a deterministic effect: the position of the plane is

8 rotated in space, or, in other words, the position of the virtual reference plane is erroneous.

9 This problem should be considered in many cases as a minor problem.

11 Now, it is interesting to quantify how the calibration errors may induce a reconstruction error

12 independently from the reference plane absolute position. Because the tests are pure 13 translation, the reconstruction error can be simply defined as the difference between the 14 current reconstructed shape and the plane fitting the field in the least square error assumption.

15 The reconstruction error is divided into two contributions: a high frequency one, representing 16 a random error, mainly related to the phase error, and a low-frequency one, related to 17 calibration uncertainties. This latter might be approximated by a quadratic function, and 18 results in an erroneous curvature. In the following, the calibration uncertainties will only be 19 characterized by a standard deviation. Calibration uncertainties represent $15 \mu \mathrm{m}$ and the 20 random noise $50 \mu \mathrm{m}$ in the studied case $(\mathrm{P}=66 \%)$. As a consequence, the calibration seems 21 sufficiently efficient, and efforts have to be put on the random phase noise. The total error on 22 the instrument is in this situation $104 \mu \mathrm{m}(\mathrm{P}=95 \%)$.

23 Last, a second test-case has been studied, corresponding to a high-sensitivity set-up: the field 24 of view has been decreased to $41 \times 31 \mathrm{~mm}^{2}$; the fringe density has been set to a maximum 25 value, considering both the camera and the video-projector resolution (8 pixels per fringe). 
1 Global geometry is the same, even if optical elements are supposed to be ten times closer.

2 Rotation angles are identical as before (see Tables 4 and 5). Last, the random noise level has

3 been decreased, considering that for high sensitivity results, a quasi-static situation may be

4 achieved and that a higher number of pictures should be taken. Noise level has been set to a

5 reasonable minimum value of $0.5 \%$ of fringe. In this situation, mean sensitivity is 0.6

$6 \mathrm{~mm} /$ fringe. The same trends are observed in this configuration, but the scale itself is

7 decreased. Random error is $3 \mu \mathrm{m}$, and bias due to mispositioning is $4 \mu \mathrm{m}(\mathrm{P}=66 \%)$. In this

8 situation, the total error on the instrument is estimated to be $10 \mu \mathrm{m}(\mathrm{P}=95 \%)$.

9 It is worth noting that the two overall error values $(10 \mu \mathrm{m}$ and $104 \mu \mathrm{m})$ correspond to the

10 experience in the laboratory, as illustrated in section 5.3. . Error is mainly determined by the

11 random error (phase error) and by the set-up sensitivity. With a high sensitivity set-up (second

12 case), the random error becomes small enough so that the calibration error becomes 13 significant.

\section{7. Summary and conclusions}

16 Error estimation on optical full field techniques (OFFT) is millstone in the diffusion of OFFT.

17 The present work describes a generic way to estimate overall error in fringe projection, either

18 due to random sources (phase error, basically related to the quality of the camera and of the

19 fringe extraction algorithm) or the bias (calibration errors). Here, a high level calibration

20 procedure based on pinhole model has been implemented. This model compensates for the

21 divergence effects of both the video-projector and the camera.

22 The work is based on a Monte Carlo procedure. So far, the complete models of the calibration

23 procedure and of a reference experiment are necessary. Here, the reference experiment

24 consists in multiple step out-of-plane displacements of a plane surface. Using this very simple 
1 test, it is possible to observe that:

2 1- The uncertainties in the calibration procedure lead to a global rotation of the plane ; this

3 means that a surface is reconstructed in a frame of reference slightly different from the global

4 frame of reference of the experimental set-up. As a matter of fact, a variation between a

5 reference position and a stressed one becomes independent of this parameter.

6 2- The overall error has been calculated in two situations: a macroscopic one, with standard

7 noise level, and a microscopic one, with a lower -but still realistic- noise level. The overall 8 error ranges from $104 \mu \mathrm{m}$ down to $10 \mu \mathrm{m}$.

9 3- The main error source is the phase error at a macroscopic level and at a microscopic level,

10 even if in this latter, errors due to the calibration are not negligible any more.

12 Results are calibration-dependent: using another calibration procedure might lead to a

13 different error distribution between calibration error and phase error. Anyway, as a generic

14 tool, the Monte-Carlo procedure has to be considered.

15 Finally, the aim of such a tool is to give some quantitative data on the overall uncertainty; this

16 work can be easily used to determine before experiments the performance of a fringe

17 projection set-up. So far, it has been proved here to be efficient to find some interesting 18 features at a microscopic level.

\section{Acknowledgements}

21 Authors thank Yunjin Kim, from Korean Advanced Institute of Science and Technology for 22 her help on this work.

\section{References}

24 [1] Schreier H.W. and Sutton M.A., Systematic Errors in Digital Image Correlation Due 25 to Undermatched Subset Shape Functions, Experimental Mechanics, 43, 3, (2002) 303-310 
1 [2] Bornert M., Brémand F., Doumalin P., Dupré J.C., Fazzini M., Grédiac M., Hild F.,

2 Mistou S., Molimard J., Orteu J.J., Robert L., Surrel Y., Vacher P., Wattrisse B., Assessment

3 of Digital Image Correlation measurement errors: Methodology and results, Experimental

4 Mechanics 49, 3 (2009) 353-370.

5 [3] Surrel Y., Additive noise effect in digital phase detection, Applied Optics, 36, 1 (1997) $6 \quad 271-276$.

7 [4] Lecompte D., Smits A., Bossuyt S., Sol H., Vantomme J., Van Hemelrijck D., Habraken

8 A.M., Quality assessment of speckle patterns for digital image correlation, Optics and Lasers 9 in Engineering, 44, 11 (2006) 1132-1145.

10 [5] Pazos V., Cheriet F., Song L., Labelle H., Dansereau J., Accuracy assessment of human 11 trunk surface 3D reconstructions from an optical digitising system, Medical and Biological 12 Engineering and Computing, 43, 1 (2005) 11-15.

13 [6] Bing Pan, Qian Kemao, Lei Huang, and Anand Asundi, Phase error analysis and 14 compensation for nonsinusoidal waveforms in phase-shifting digital fringe projection 15 profilometry, Optics Letter, 34, 4 (2009) 416-418.

16 [7] Cordero R., Molimard J., Martinez A., Labbé F., Uncertainty Analysis of Temporal Phase-

17 Stepping Algorithms for Interferometry, Optics Communications, 275 (2007)144-155.

18 [8] Cordero R.R., Molimard J., Labbé F., Martínez A., Strain maps obtained by phase-shifting 19 interferometry: An uncertainty analysis, Optics Communications, 281 (2008) 2195-2206.

20 [9] Cordero R.R., Martınez A., Rodrıguez-Vera R., Roth P., Uncertainty evaluation of 21 displacements measured by electronic speckle-pattern interferometry, Optics Communications $22 \quad 241(2004) 279-292$

23 [10] Benoit P., Mathieu E., Hormière, J. Thomas A., Characterization and control of three- 
1 dimensional objects using fringe projection techniques, Nouvelle Revue d'Optique 6, 2 (1975)

267.

3 [11] Takeda M., Ina H., Kobayashi S., Fourier-transform method of fringe-pattern analysis for

4 computer-based topography and interferometry, Journal of the Optical Society of America,

$5 \quad 72,1(1982)$ 156-160.

6 [12] Gorthi S.S., Rastogi P., Fringe projection techniques: wither we are?, Optics and Lasers

7 in Engineering, 48, 2 (2010) 133-140.

8 [13] Gigliotti M., Molimard J., Jacquemin F., Vautrin A., On the nonlinear deformations of

9 thin unsymmetric 0/90 composite plates under hygrothermal loads, Composites Part A:

10 Applied Science and Manufacturing, 37, 4 (2006) 624-629.

11 [14] Nam Nguyen T., M Huntley J., Burguete R., Russell Coggrave C., Combining digital

12 image correlation and projected fringe techniques on a multi-camera multi-projector platform,

13 Journal of Physics: Conference Series, 181 (2009) 012076 (8pp).

14 [15] Molimard J., Boyer G., Zahouani H., Frequency-based image analysis of random 15 patterns: an alternative way to classical stereocorrelation, Journal of the Korean society of non 16 destructive testing, 30, 3 (2010) 181-193.

17 [16] Peisen S. Huang, Chengping Zhang, and Fu-Pen Chiang, High-speed 3-D shape 18 measurement based on digital fringe projection, Optical Engineering, 42 (2003) 163-168.

19 [17] Lagarde J.M., Rouvrais C., Black D., Diridollou S., Gall Y., Skin topography 20 measurement by interference fringe projection: a technical validation, Skin Res Technol., 7, 2 21 (2001) $112-121$.

22 [18] Breque C., Dupre J.C., Bremand F., Calibration of a system of projection moiré for relief 23 measuring: biomechanical applications, Optics and Lasers in Engineering, 41, 2 (2004) 241- 
1260.

2 [19] Zahouani H., Vargiolu R., Skin morphology and volume: methods of evaluation, in

3 Injection treatments in cosmetic surgery, Ascher B. and al. ed., Informa, London 2009, 20 p.

4 [20] Martínez A., Rayas J.A., Cordero R.R., Balieiro D., Labbe F., Leaf cuticle topography

5 retrieved by using fringe projection, Optics and Lasers in Engineering, 50, 2 (2012) 231-235

6 [21] Sciammarella C.A., Lamberti L., Sciammarella F.M., High-accuracy contouring using

7 projection moiré, Optical Engineering, 44, 9 (2005) 093605 (12pp.).

8 [22] ISO 2004 Guide to the Expression of Uncertainty in Measurement, Supplement 1:

9 Numerical Methods for the Propagation of Distributions (Geneva: ISO) p 38

10 [23] Cordero R. Roth P., On Two Methods to Evaluate the Uncertainty of Derivatives

11 Calculated from Polynomials Fitted to Experimental Data, Metrologia, 42 (2005) 39-44

12 [24] Hall B., Computer modelling of uncertainty calculations with finite degrees of freedom,

13 Meas Sci Technol, 112000 1335-41

14 [25] ISO 1993 Guide to the Expression of Uncertainty in Measurement (Geneva: ISO) p 101

15 [26] Lira I, Evaluating the Uncertainty of Measurement: Fundamentals and Practical

16 Guidance (Bristol: Institute of Physics Publishing), 2002, p 239

17 [27] Chen Xiaobo, Xi Jun tong, Jiang Tao, Jin Ye, Research and development of an accurate

18 3D shape measurement system based on fringe projection: Model analysis and performance

19 evaluation, Precision Engineering, 32, 3 (2008) 215-221

20 [28] Sutton MA, Orteu JJ, Schreier HW, Image correlation for shape, motion and

21 deformation measurements: basic concepts, theory and applications, Springer, New-York, 222009,332 p. 
1 [29] Surrel Y., Moiré and grid methods in optics, SPIE 2342 (1994) 213-220.

2 [30] Wang P., Drapier S., Molimard J., Vautrin A., Minni J.C., Characterization of Liquid 3 Resin Infusion (LRI) filling by fringe pattern projection and in situ thermocouples, 4 Composites Part A: Applied Science and Manufacturing, 41, 1 (2010) 36-44.

5 [31] Bouguet J.Y., Camera Calibration Toolbox for Matlab. http://www.vision.caltech.edu/ 6 bouguetj/calib_doc/. 


\section{List of tables}

2 Table 1. Reference shape measurement: overall uncertainties of a fringe projection set-up.

3 Table 2. Geometrical parameters and uncertainties (macroscopic scale).

4 Table 3. Calibration parameters and uncertainties (macroscopic scale).

5 Table 4. Geometrical parameters and uncertainties (microscopic scale).

6 Table 5. Calibration parameters and uncertainties (microscopic scale). 


\section{List of figures}

2 Figure 1: Illustration of the pin-hole model.

3 Figure 2. Optical set-up and calibration test-rig.

4 Figure 3. Fringe projection basic principle.

5 Figure 4. Fringe projection calibration.

6 Figure 5. Phase map error. a/ without correction b/ after correction c/ PDF of row map ( $\sigma=$

$7 \quad 0.3 \times 10-2 \times 2 \pi) \mathrm{d} / \mathrm{PDF}$ of corrected map $(\sigma=0.2 \times 10-2 \times 2 \pi)$.

8 Figure 6. Reconstruction of a reference sphere-in-plate.

9 Figure 7. Bias on plane $\mathrm{z}=0 \mathrm{~mm}$.

10 Figure 8. Random error on plane $\mathrm{z}=0 \mathrm{~mm}$. 


\begin{tabular}{|c|c|c|}
\hline & Value & Resolution \\
\hline Dial indicator & $1.93 \mathrm{~mm}$ & $14 \mu \mathrm{m}$ \\
\hline Fringe projection & $2.1 \mathrm{~mm}$ & $43 \mu \mathrm{m}$ \\
\hline
\end{tabular}

Table 1. Reference shape measurement: overall uncertainties of a fringe projection setup. 


\begin{tabular}{ccccc}
\hline & $\mathrm{P}_{\mathrm{t}} / \mathrm{f}_{\mathrm{p}}(\mathrm{m} / \mathrm{m})$ & $\mathrm{h}_{\mathrm{p}}(\mathrm{mm})$ & $\mathrm{h}_{0}(\mathrm{~mm})$ & $\mathrm{d}(\mathrm{mm})$ \\
\hline Reference value & $5.1 \times 10^{-3}$ & -474 & -340 & 341 \\
Uncertainty & $7 \times 10^{-4}$ & 2 & 2 & 2 \\
Error type & B & B & B & B
\end{tabular}

Table 2. Geometrical parameters and uncertainties (macroscopic scale). 


\begin{tabular}{|c|c|c|c|c|}
\hline Parameters & PDF & $\begin{array}{l}\text { Nominal } \\
\text { value }\end{array}$ & $\begin{array}{c}1 / 2 \text { length or } \\
\text { standard } \\
\text { deviation }\end{array}$ & Error type \\
\hline
\end{tabular}

Calibration parameters

$\begin{array}{ccccc}\alpha & \text { uniform } & 2.1^{\circ} \text { to } 4.2^{\circ} & 0.14^{\circ} & \text { B } \\ \gamma_{\mathrm{CCD}} & \text { gaussian } & 53 \mu \mathrm{m} & 0.06 \mu \mathrm{m} & \mathrm{B} \\ \tau_{\mathrm{CCD}} & \text { gaussian } & 1 & 0.017 & \mathrm{~B}\end{array}$

Reference plane mispositionning

$\begin{array}{ccccc}\beta_{1}, \beta_{2}, \beta_{3} & \text { gaussian } & 0 & 3^{\prime} & B \\ \mathrm{x}_{1}, \mathrm{x}_{2}, \mathrm{x}_{3} & \text { gaussian } & 0 & 0.25 \mathrm{~mm} & \mathrm{~B}\end{array}$

Phase dispersion

$\delta \varphi \quad$ gaussian $\quad 0 \quad 10^{-2} \times 2 \pi \quad \mathrm{A}$

Table 3. Calibration parameters and uncertainties (macroscopic scale). 


\begin{tabular}{ccccc}
\hline & $\mathrm{P}_{\mathrm{t}} / \mathrm{f}_{\mathrm{p}}(\mathrm{m} / \mathrm{m})$ & $\mathrm{h}_{\mathrm{p}}(\mathrm{mm})$ & $\mathrm{h}_{0}(\mathrm{~mm})$ & $\mathrm{d}(\mathrm{mm})$ \\
\hline Reference value & $5.1 \times 10^{-3}$ & -285 & -197 & 205 \\
Uncertainty & $1 \times 10^{-3}$ & 1 & 1 & 1 \\
Error type & B & B & B & B
\end{tabular}

Table 4. Geometrical parameters and uncertainties (microscopic scale). 


\begin{tabular}{|c|c|c|c|c|}
\hline Parameters & PDF & $\begin{array}{l}\text { Nominal } \\
\text { value }\end{array}$ & $\begin{array}{l}\text { 1/2 length or } \\
\text { standard } \\
\text { deviation }\end{array}$ & Error type \\
\hline
\end{tabular}

Calibration parameters

$\begin{array}{ccccc}\alpha & \text { uniform } & 2.1^{\circ} \text { to } 4.2^{\circ} & 0.14^{\circ} & \text { B } \\ \gamma_{\mathrm{CCD}} & \text { gaussian } & 32 \mu \mathrm{m} & 0.036 \mu \mathrm{m} & \mathrm{B} \\ \tau_{\mathrm{CCD}} & \text { gaussian } & 1 & 0.017 & \mathrm{~B}\end{array}$

Reference plane mispositionning

$\begin{array}{ccccc}\beta_{1}, \beta_{2}, \beta_{3} & \text { gaussian } & 0 & 2^{\prime} & B \\ \mathrm{x}_{1}, \mathrm{x}_{2}, \mathrm{x}_{3} & \text { gaussian } & 0 & 0.16 \mathrm{~mm} & \mathrm{~B}\end{array}$

Phase dispersion

$\delta \varphi \quad$ gaussian $\quad 0 \quad 0.5 \times 10^{-2} \times 2 \pi \quad \mathrm{A}$

Table 5. Calibration parameters and uncertainties (microscopic scale). 


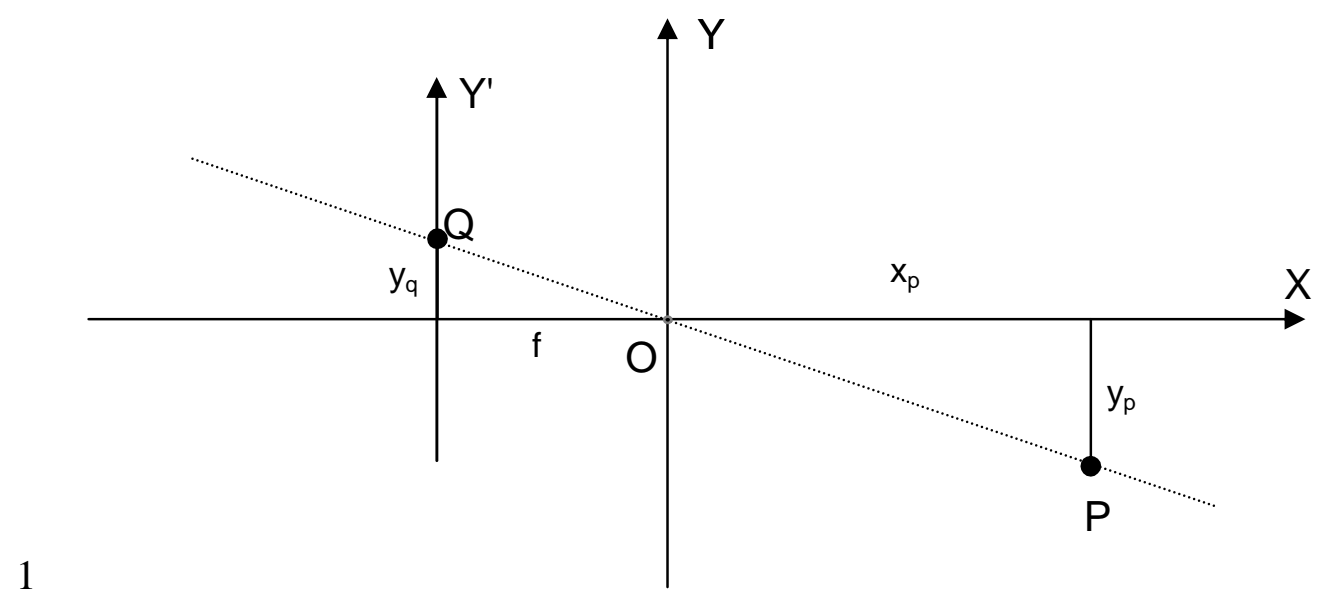

Figure 1: Illustration of the pin-hole model.

3 


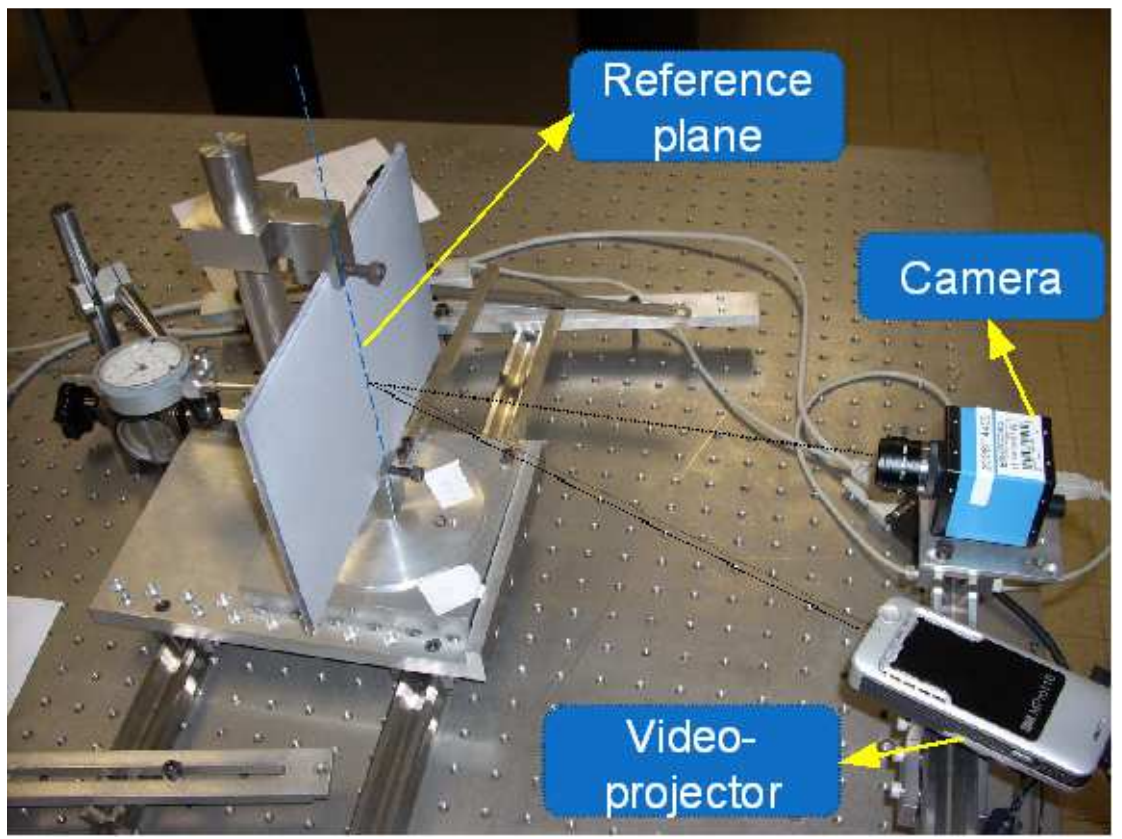

Figure 2. Optical set-up and calibration test-rig. 


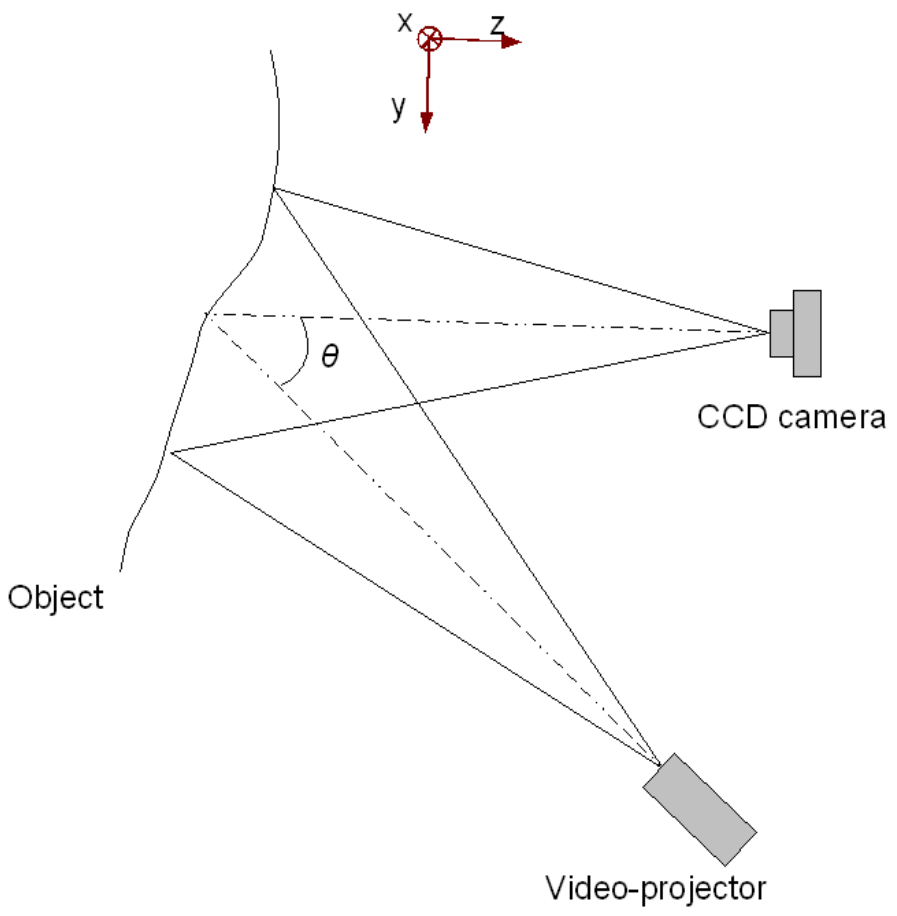

Figure 3. Fringe projection basic principle. 

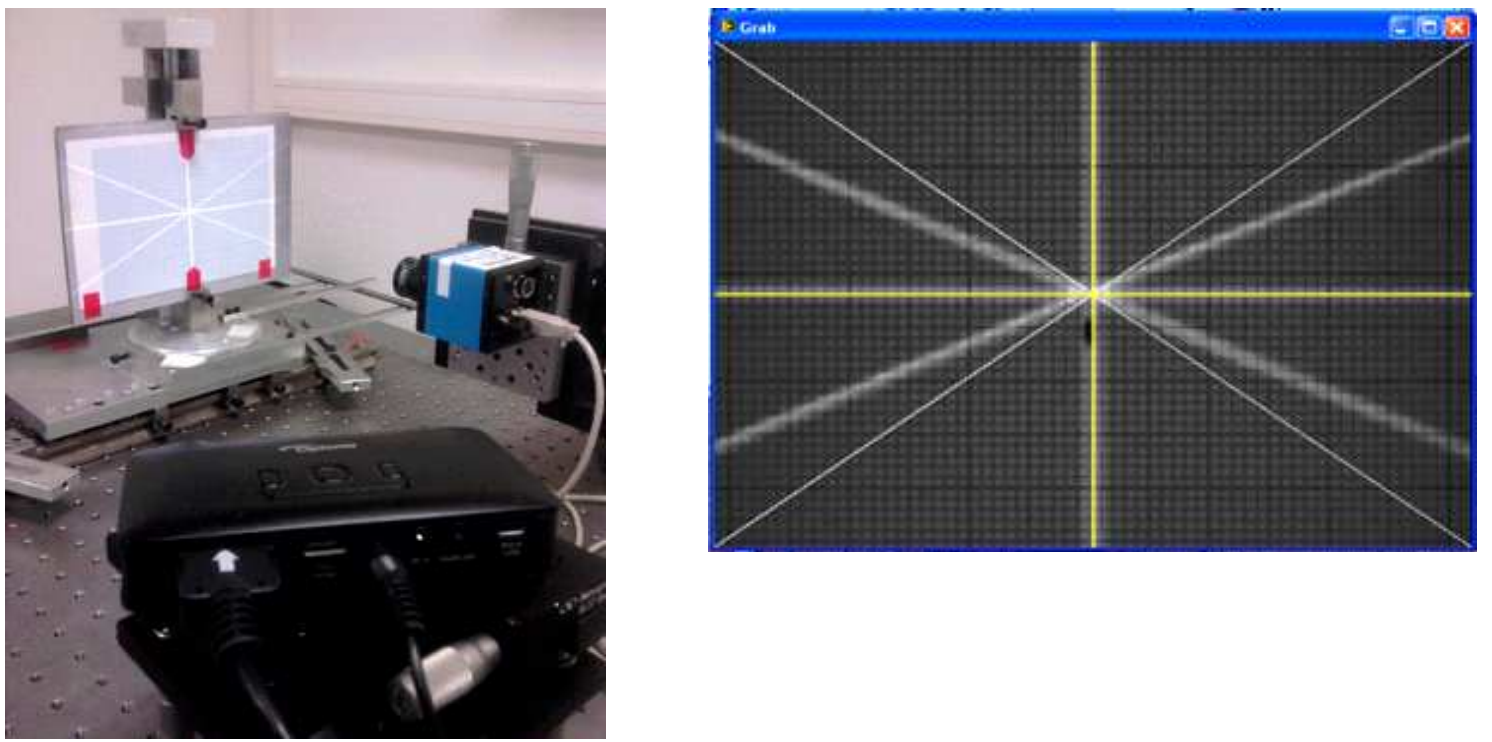

Figure 4. Fringe projection calibration. 

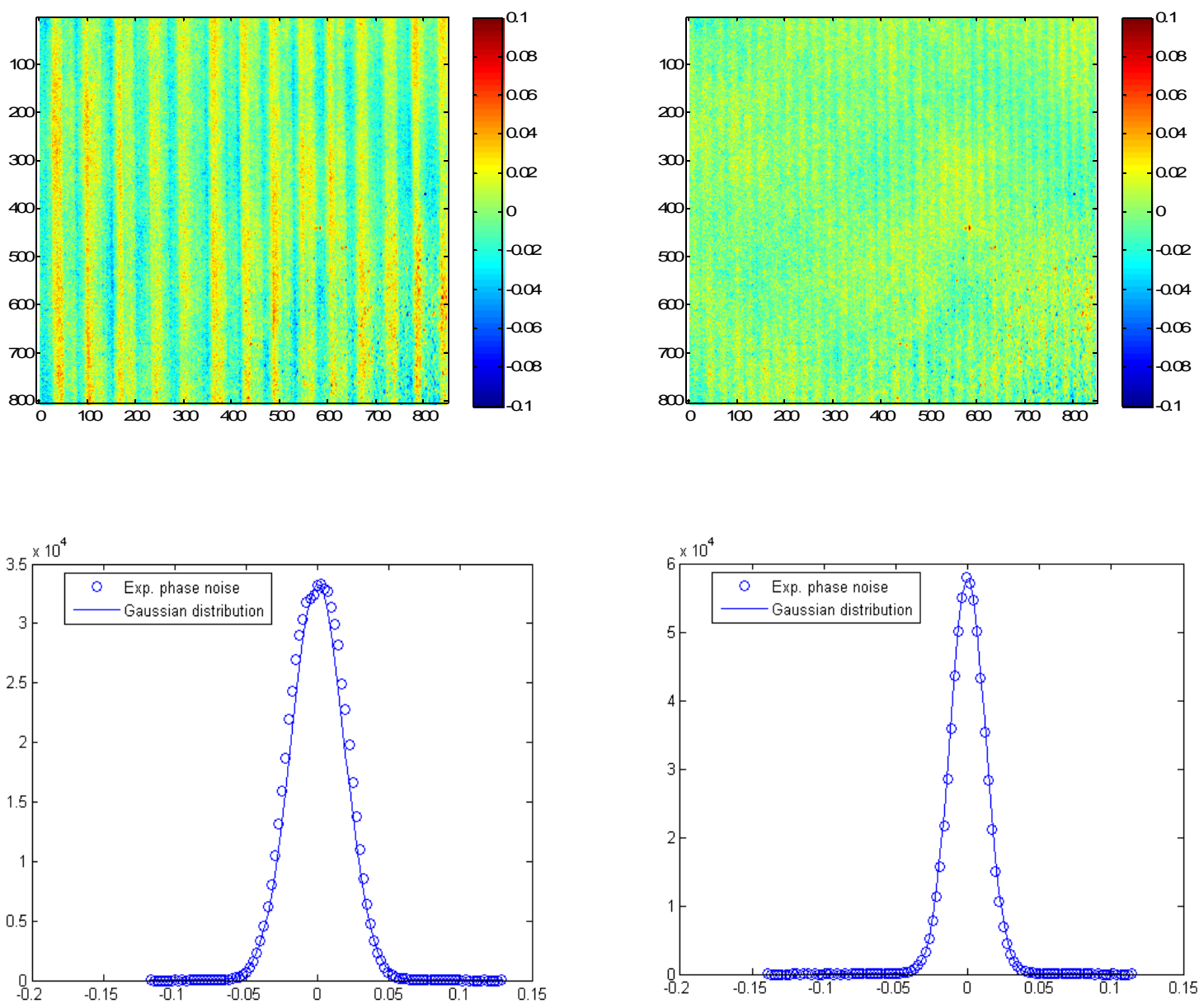

Figure 5. Phase map error. a/ without correction b/ after correction c/ PDF of row map $\left(\sigma=0.3 \times 10^{-2} \times 2 \pi\right) \mathrm{d} / \mathrm{PDF}$ of corrected map $\left(\sigma=0.2 \times 10^{-2} \times 2 \pi\right)$. 


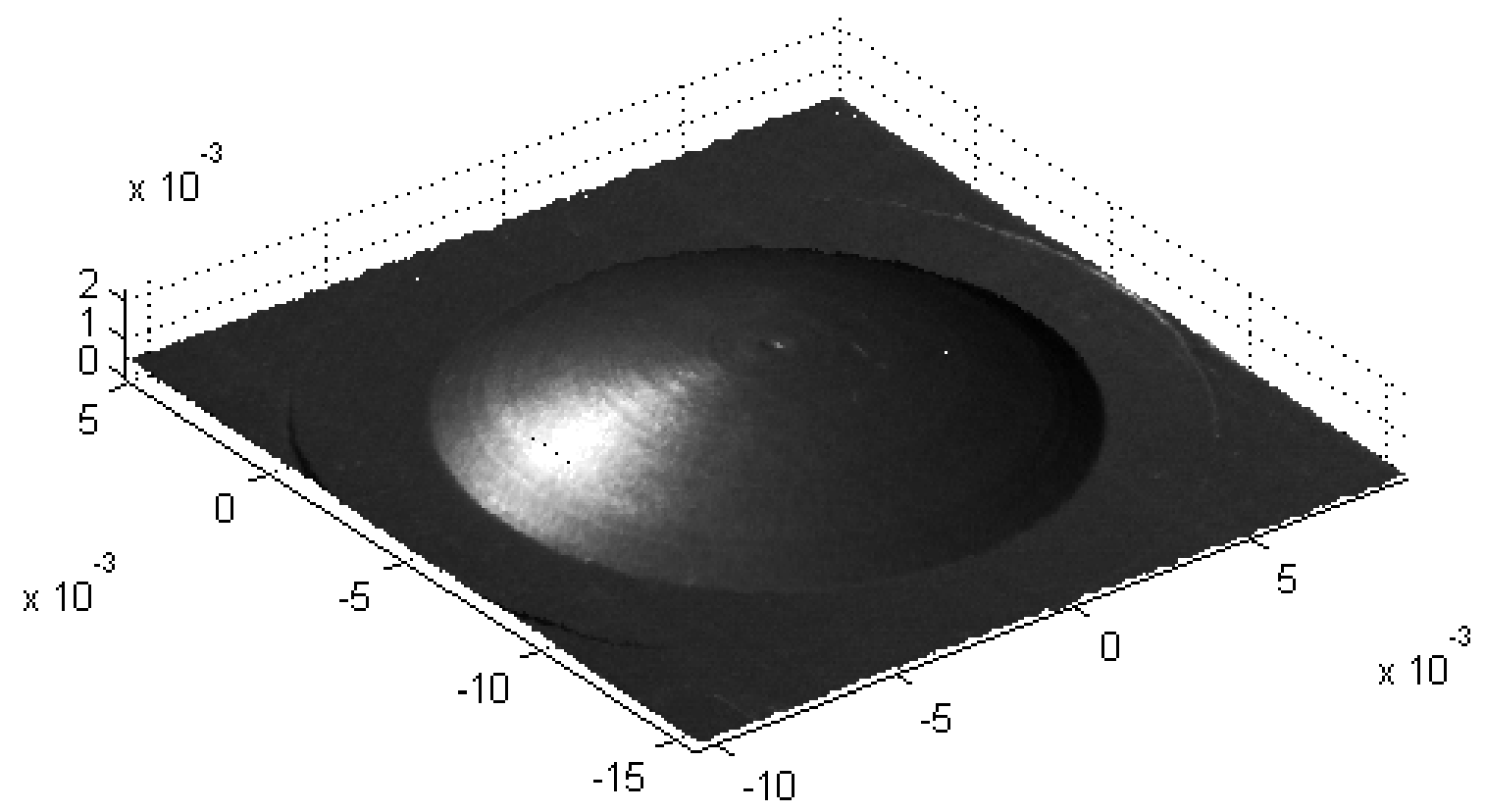

Figure 6. Reconstruction of a reference sphere-in-plate. 


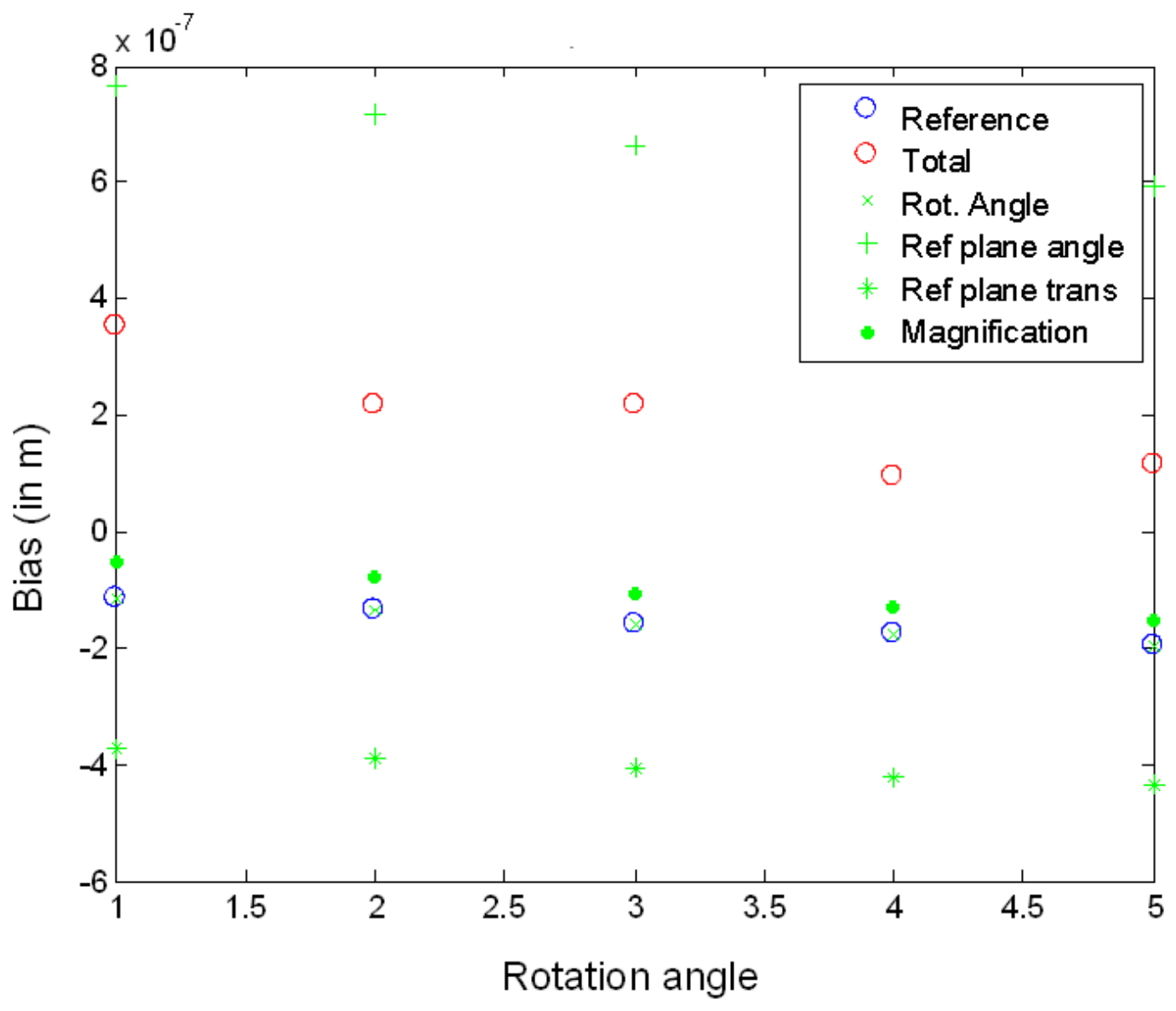

Figure 7. Bias on plane $z=0 \mathrm{~mm}$. 


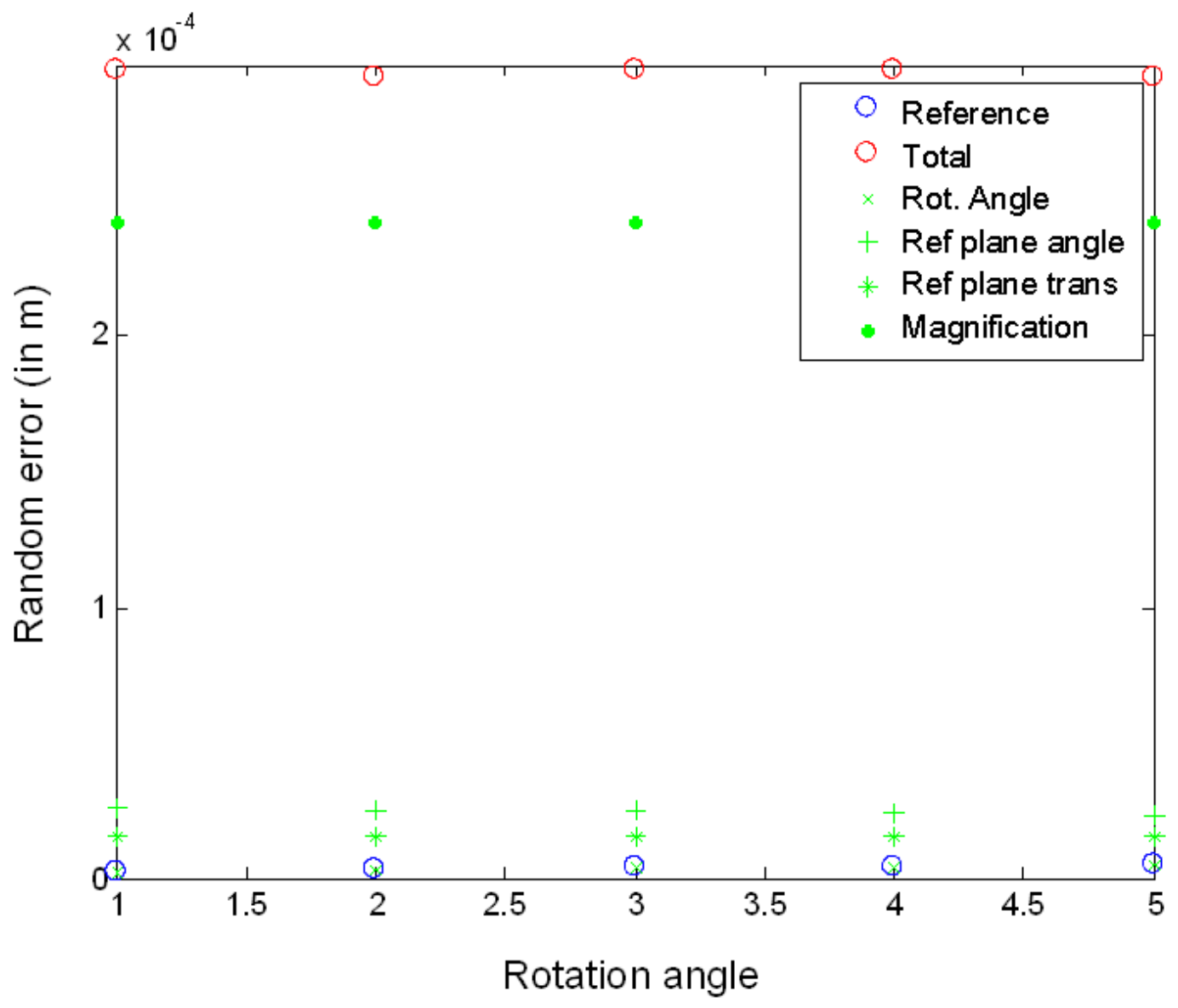

Figure 8. Random error on plane $z=0 \mathrm{~mm}$. 